International Journal of Pure and Applied Mathematics

Volume 89 No. $1 \quad 2013,1-7$

ISSN: 1311-8080 (printed version); ISSN: 1314-3395 (on-line version)

url: http://www.ijpam.eu

doi: http://dx.doi.org/10.12732/ijpam.v89i1.1

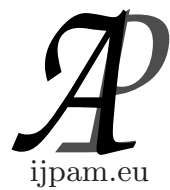

\title{
FOUR-DIMENSIONAL RIEMANNIAN STANILOV-VIDEV MANIFOLDS
}

\author{
Maria Ivanova ${ }^{1}$, Veselin Videv $^{2} \S$ \\ Metropolitan State University of Denver \\ Mathematical and Computer Sciences \\ P.O. Box 173362, Campus Box 38, Denver, Colorado 80217-3362, USA \\ Department of Mathematics and Informatics \\ Trakia University \\ St. Zagora, 6000, BULGARIA
}

\begin{abstract}
In the present note we characterize the four-dimensional Riemannian manifolds for which any skew-symmetric Stanilov operator with respect to any plane in the tangent space to the manifold commute with the corresponding generalized Jacobi operator, at any point of the manifold. These classes of manifolds Gilkey called Stanilov-Videv manifolds.
\end{abstract}

AMS Subject Classification: 53C20, 53C25

Key Words: Riemannian manifold, Jacobi operator, skew-symmetric Stanilov operator, generalized Jacobi operator, commutative conditions, Stanilov-Videv manifold

Let $(M, g)$ be an $n$-dimensional Riemannian manifold with a metric tensor $g$ and curvature tensor $R$, let $p$ be a point of $M$, and let $p$ be the tangent space to the manifold at this point. Then we can consider the following curvature operators:

Received: January 11, 2013

(C) 2013 Academic Publications, Ltd. url: www.acadpubl.eu

${ }^{\S}$ Correspondence author 


$$
\text { Jacobi operator: } \quad R_{X}(u)=R(u, X, X),
$$

defined for any tangent vector $X \in M_{p}$, at any point $p \in M$ (see [1]),

$$
\text { Skew-symmetric Stanilov operator: } \quad S_{\alpha}(u)=S_{X, Y}(u)=R(X, Y, u),
$$

defined for an arbitrary orthonormal basis $X, Y$ in the plane $\alpha \in M_{p}$, at a point $p \in M($ see $[4])$,

$$
\text { Generalized Jacobi operator: } \quad R_{\pi}(u)=\sum_{i=1, m} R_{X i}(u),
$$

defined for an arbitrary $m$ - dimensional tangent subspace $\pi \subset M_{p}$, at a point $p \in M$, where $\left\{X_{i}\right\}_{i=1, m}(m<n)$ is an orthonormal basis in $\pi$, see [2].

Having in mind our preliminary results [7], Peter Gilkey introduces the following definitions [3]:

Definition 1. An $n$-dimesional Riemannian manifold $(M, g)$ is StanilovVidev manifold if for any two-dimensional plane $\alpha \in M_{p}$, at any point $p \in M$, the following equality holds:

$$
S_{\alpha} \circ R_{\alpha}=R_{\alpha} \circ S_{\alpha}
$$

Further in the present paper we'll characterize the four-dimensional StanilovVidev manifolds. First we replace the commutative condition (1) with the equivalent conditions $S_{\alpha} \circ R_{\alpha}$ to be a skew-symmetric curvature operator and we will show that if $(1)$ holds, then $(M, g)$ is an Einstein Riemannian manifold, which means that (see $[6])$ :

$$
\rho=c g,
$$

where $c$ is a constant and $\rho$ is the Ricci tensor on the manifold $(M, g)$.

Let $\left\{e_{1}, e_{2}, e_{3}, e_{4}\right\}$ be an orthonormal basis in the tangent space $M_{p}$, at a point $p \in M$. If $\alpha$ is a two-dimensional plane, spanned from the tangent vectors $e_{1}$ and $e_{2}$, then:

$$
\begin{aligned}
\left(S_{\alpha}\right) & =\left(\begin{array}{llll}
0 & -K_{12} & -R_{2113} & -R_{2114} \\
K_{12} & 0 & R_{1223} & R_{1224} \\
R_{2113} & -R_{1223} & 0 & R_{1234} \\
R_{2114} & -R_{1224} & -R_{1234} & 0
\end{array}\right), \\
\left(R_{\alpha}\right) & =\left(\begin{array}{llll}
K_{12} & 0 & R_{1223} & R_{1224} \\
0 & K_{12} & R_{2113} & R_{2114} \\
R_{1223} & R_{2113} & K_{13}+K_{23} & R_{3114}+R_{3224} \\
R_{1224} & R_{2114} & R_{3114}+R_{3224} & K_{14}+K_{24}
\end{array}\right)
\end{aligned}
$$


From here, multiplying, for the entries of the operator matrix $A:=S_{\alpha} \circ R_{\alpha}$, with respect to the orthonormal basis $\left\{e_{1}, e_{2}, e_{3}, e_{4}\right\}$, we obtain:

$$
\begin{aligned}
& a_{11}=-R_{2113} R_{1223}-R_{2114} R_{1224}, \\
& a_{22}=R_{1223} R_{2113}+R_{1224} R_{2114}, \\
& a_{33}=R_{1234}\left(R_{3114}+R_{3224}\right), \\
& a_{44}=-R_{1234}\left(R_{3114}+R_{3224}\right), \\
& a_{12}=-K_{12}^{2}-R_{2113}^{2}-R_{2114}^{2}, \\
& a_{21}=K_{12}^{2}+R_{1223}^{2}+R_{1224}^{2}, \\
& a_{13}=-K_{12} R_{2113}-\left(K_{13}+K_{23}\right) R_{2113}-R_{2114}\left(R_{3114}+R_{2114}\right), \\
& a_{31}=K_{12} R_{2113}+R_{1234} R_{1224}, \\
& a_{14}=-K_{12} R_{2114}-R_{2113}\left(R_{3114}+R_{3224}\right)-R_{2114}\left(K_{14}+K_{24}\right), \\
& a_{41}=K_{12} R_{2114}-R_{1234} R_{1223}, \\
& a_{23}=\left(K_{12}+K_{13}+K_{23}\right) R_{1223}+R_{1224}\left(R_{3114}+R_{3224}\right), \\
& a_{32}=-K_{12} R_{1223}+R_{2114} R_{1234}, \\
& a_{24}=K_{12} R_{1224}+R_{1223}\left(R_{3114}+R_{3224}\right)+\left(K_{14}+K_{24}\right) R_{1224}, \\
& a_{42}=-K_{12} \cdot R_{1224}-R_{1234} R_{2113}, \\
& a_{34}=R_{2113} R_{1224}-R_{1223} R_{2114}+\left(K_{14}+K_{24}\right) R_{1234}, \\
& a_{43}=R_{2114} R_{1223}-R_{1224} R_{2113}-R_{1234}\left(K_{13}+K_{23}\right) .
\end{aligned}
$$

Since $A$ is a skew-symmetric matrix, then we have the equalities:

$$
a_{i i}=0, \quad a_{i j}=-a_{j i},
$$

for any indices $i, j=1,2,3,4$, and using more exactly that $a_{12}=-a_{21}$, according to the respective expression in (3), we obtain the equality:

$$
R_{2113}^{2}+R_{2114}^{2}=R_{1223}^{2}+R_{1224}^{2} .
$$

Let we choose $\left\{e_{1}, e_{2}, e_{3}, e_{4}\right\}$ to be an orthonormal eigenvector basis of the Jacobi operator $R_{e 1}$. Then from this condition we obtain that:

$$
R_{2113}=R_{2114}=R_{3114}=0 .
$$

Now from (5) and (6) we get:

$$
R_{2113}=R_{2114}=R_{1223}=R_{1224}=0 .
$$


Since the hypothesis (1) is true for any plane $\alpha=e_{i} \wedge e_{j}$, similarly to the way of obtaining (7), we get the following equalities:

$$
\begin{aligned}
& R_{2113}=R_{2114}=R_{1223}=R_{1224}=R_{3112}=R_{3114}=R_{1332} \\
& \quad=R_{1334}=R_{4112}=R_{4113}=R_{1442}=R_{1443}=0 .
\end{aligned}
$$

From this we conclude that for the entries of the Ricci tensor is true

$$
\rho_{12}=\rho_{13}=\rho_{14}
$$

which in turn gives us

$$
\rho_{1 x}=\rho\left(e_{1}, x\right)=0,
$$

for any unit tangent vector $x \in M_{p}$, such that $x \perp e_{1}$, at any point $p \in M$. This result according to the Herglotz theorem means that $(M, g)$ is a fourdimensional Einstein Riemannian manifold [6], that was our result in [7], and in the sequel we will continue these investigations to prove main result in the present paper.

Suppose again that $\left\{e_{1}, e_{2}, e_{3}, e_{4}\right\}$ is an arbitrary orthonormal basis in the tangent space $M_{p}$, at a point $p \in M$. Then from the hypothesis (1) we have the equality $a_{13}=-a_{31}$ and from the respective expressions in (3) we get the equation:

$$
\left(K_{13}+K_{23}\right) R_{2113}+R_{2114}\left(R_{3114}+R_{2114}\right)=R_{1234} R_{1224} .
$$

Since $(M, g)$ is an Einstein Riemannian manifold, then $K_{13}=K_{23}$, and now the last equality gives us:

$$
\left(K_{13}+K_{14}\right) R_{2113}+R_{2114} R_{3114}+R_{2114}^{2}=R_{1234} R_{1224} .
$$

Since here $\left\{e_{1}, e_{2}, e_{3}, e_{4}\right\}$ is an orthonormal basis in the tangent space $M_{p}$, at an arbitrary point $p \in M$, then we can substitute $e_{2}$ with $-e_{2}$. In this way we obtain the following equality:

$$
-\left(K_{13}+K_{14}\right) R_{2113}-R_{2114} R_{3114}+R_{2114}^{2}=-R_{1234} R_{1224}
$$

Summing (8) and (9), we obtain the equation:

$$
R_{2114}^{2}=0 \text {. }
$$

From the last equality it follows that

$$
R(x, y, y, z)=0
$$


for an arbitrary orthonormal triple of tangent vectors $x, y, z \in M_{p}$, at any point $p \in M$, because of $\left\{e_{1}, e_{2}, e_{3}, e_{4}\right\}$ is an orthonormal basis in the tangent space $M_{p}$. That means that we can apply the last equality for the orthonormal triple of tangent vectors $\left\{\frac{x+z}{\overline{2}}, y, y, \frac{x \neq z}{\overline{2}}\right\}$ and so we get the equation:

$$
R\left(\frac{x+z}{\sqrt{2}}, y, y, \frac{x-z}{\sqrt{2}}\right)=0
$$

from which it follows that

$$
R(x+z, y, y, x-z)=0 .
$$

This equality is equivalent to the equation:

$$
K(x, y)=K(y, z),
$$

for any orthonormal triple of tangent vectors $x, y, z \in M_{p}$, at any point $p \in M$, where $K$ is a function of the sectional curvature of the manifold $(M, g)$.

Since we proved above that $(M, g)$ is an Einstein four-dimensional manifold, then at any point $p \in M$ there exists an orthonormal tangent Singer-Thorpe basis $\left\{e_{1}, e_{2}, e_{3}, e_{4}\right\}$ in the tangent space $M_{p}$, with respect to which the curvature tensor entries satisfy the following equalities (see [5]):

$$
\begin{gathered}
K_{12}=K_{34}=\lambda_{1}, \quad K_{13}=K_{24}=\lambda_{2}, \quad K_{14}=K_{23}=\lambda_{3}, \\
R_{1234}=\mu_{1}, \quad R_{1342}=\mu_{2}, \quad R_{1423}=\mu_{3}, \\
R_{1223}=R_{1443}=R_{1332}=R_{1442}=R_{1224}=R_{1334}=0,
\end{gathered}
$$

where

$$
\mu_{1}+\mu_{2}+\mu_{3}=0, \quad \lambda_{1}+\lambda_{2}+\lambda_{3}=\frac{\tau}{4},
$$

$\tau$ is a scalar curvature of $(M, g)$ and $\lambda_{1}=\max K, \lambda_{3}=\min K$. From here and (10) we get that $\max K=\min K$, which means that $K$ is a constant at any point $p \in M$, which according to the Shour's theorem means that $K$ is a global constant of the manifold $(M, g)$, see [6]. Then according to definition $(M, g)$ is a four-dimensional Riemannian manifold of a constant sectional curvature and curvature tensor of the form

$$
R(x, y, z)=K(g(y, z) x-g(x, z) y), K=\text { const. }
$$

for any tangent vectors $x, y, z \in M_{p}$, at any point $p \in M$, see [6]. 
Conversely, if $(M, g)$ is a four-dimensional Riemannian manifold of a constant sectional curvature and curvature tensor of the form (11), then for any two-dimensional tangent plane $\alpha \in M_{p}$, at any pont $p \in M$, and for any orthonormal basis $\left\{e_{1}, e_{2}, e_{3}, e_{4}\right\} \in M_{p}$, we have the following curvature matrix

$$
S_{\alpha}=\left(\begin{array}{llll}
0 & -c^{2} & 0 & 0 \\
c^{2} & 0 & 0 & 0 \\
0 & 0 & 0 & 0 \\
0 & 0 & 0 & 0
\end{array}\right), \quad R_{\alpha}=\left(\begin{array}{llll}
c & 0 & 0 & 0 \\
0 & c & 0 & 0 \\
0 & 0 & 2 c & 0 \\
0 & 0 & 0 & 2 c
\end{array}\right)
$$

From here we conclude that $S_{\alpha} \circ R_{\alpha}=R_{\alpha} \circ S_{\alpha}$, since these operators have the only one matrix:

$$
\left(\begin{array}{llll}
0 & -c^{3} & 0 & 0 \\
c^{3} & 0 & 0 & 0 \\
0 & 0 & 0 & 0 \\
0 & 0 & 0 & 0
\end{array}\right)
$$

Thus we have proved our main result:

Theorem 1. A four-dimensional Riemannian manifold $(M, g)$ is StanilovVidev manifold if and only if $(M, g)$ is a four-dimensional Riemannian space of constant sectional curvature.

In the pseudo-Euclidean geometry the things are different because of Gilkey proved that there exist pseudo-Riemannian Stanilov-Videv manifolds which are not pseudo-Euclidean manifolds of constant sectional curvature, see [3].

\section{References}

[1] P. Gilkey, A. Swann, L. Vanheke, Isoparametric geodesic spheres and a conjecture of Osserman cocerning the Jacobi operator, Quart. J. Math., Oxford (1995), 299-320.

[2] P. Gilkey, G. Stanilov, V.Videv, Pseudo-Riemannian manifolds whose generalized Jacobi operator has a constant characteristic polynomial, Journal of Geometry, Basel, 62 (1998), 144-153.

[3] P. Gilkey, The Geometry of Curvature Homogeneous Pseudo-Riemannian manifolds, Word Scientific Publishing Co (2010).

[4] R. Ivanova, G. Stanilov, A skew-symmetric curvature operator in Riemannian geometry, In: Symposia Gaussiana, Conf. A (Eds.: Behara, Fritsch, Lintz), Berlin, New York (1995), 391-395. 
[5] I. Singer, J. Thorpe, The curvature of 4-dimensional Einstein space, Global Analisys. Papers in Honour of K. Kodaira, University of Tokio Press, Princeton University Press (1969).

[6] G. Stanilov, Differential Geometry. Sofia, Nauka i Izkustvo, 1998.

[7] G. Stanilov, V. Videv, On the commuting of curvature operators, Mathematics and Education in Mathematics (2004), 176-180. 
